\title{
Multiphoton Double Ionization of Ar and Ne Close to Threshold
}

\author{
Yunquan Liu, ${ }^{1,2, *}$ Difa Ye, ${ }^{3,4}$ Jie Liu, ${ }^{3,4, \dagger}$ A. Rudenko, ${ }^{1,5}$ S. Tschuch, ${ }^{1}$ M. Dürr, ${ }^{1}$ M. Siegel, ${ }^{6}$ U. Morgner, ${ }^{6}$ \\ Qihuang Gong, ${ }^{2, \$}$ R. Moshammer, ${ }^{1}$ and J. Ullrich ${ }^{1, \S}$ \\ ${ }^{1}$ Max-Planck-Institut für Kernphysik, D-69117 Heidelberg, Germany \\ ${ }^{2}$ Department of Physics and State Key Laboratory for Mesoscopic Physics, Peking University, \\ Beijing 100871, People's Republic of China \\ ${ }^{3}$ Center for Applied Physics and Technology, Peking University, 100084 Beijing, People's Republic of China \\ ${ }^{4}$ Institute of Applied Physics and Computational Mathematics, 100088 Beijing, People's Republic of China \\ ${ }^{5}$ Max-Planck Advanced Study Group at CFEL, 22607 Hamburg, Germany \\ ${ }^{6}$ Leibniz Universität Hannover, Welfengarten 1, D-30167 Hannover, Germany \\ (Received 17 November 2009; revised manuscript received 15 March 2010; published 28 April 2010)
}

In kinematically complete studies we explore double ionization (DI) of $\mathrm{Ne}$ and $\mathrm{Ar}$ in the threshold regime $\left(I>3 \times 10^{13} \mathrm{~W} / \mathrm{cm}^{2}\right)$ for $800 \mathrm{~nm}, 45 \mathrm{fs}$ pulses. The basic differences are found in the twoelectron momentum distributions - "correlation" (CO) for Ne and "anticorrelation" (ACO) for Ar-that can be partially explained theoretically within a 3D classical model including tunneling. Transverse electron momentum spectra provide insight into "Coulomb focusing" and point to correlated nonclassical dynamics. Finally, DI threshold intensities, $\mathrm{CO}$ as well as ACO regimes are predicted for both targets.

DOI: 10.1103/PhysRevLett.104.173002

PACS numbers: $32.80 . \mathrm{Rm}, 32.80 . \mathrm{Fb}$

Nonsequential double ionization (NSDI) [1] of atoms and molecules in strong infrared laser fields is responsible for the dramatic enhancement of doubly charged ion yields at moderate intensities (see, e.g., [2]). Thus, NSDI represents a prominent and outstanding manifestation of the importance and subtleties of electron-electron correlations in strong fields and has continued to attract utmost attention, theoretically as well as experimentally. Still, however, the issue is not settled and it is the subject of intense debate. For example, at low intensities below the recollision threshold, where one enters the multiphoton ionization (MPI) regime, anticorrelation between the electrons has been observed recently [3] and new ionization mechanisms have been suggested [4]. The double ionization (DI) threshold itself is not established and, moreover, theory so far has concentrated just on He, leaving experimentally observed atomic structure dependences widely unexplored.

In this Letter we investigate electron correlation in NSDI and its target-species dependence for $\mathrm{Ne}$ and $\mathrm{Ar}$ at the lowest intensities ever addressed, close to the threshold. In kinematically complete measurements we find substantially different correlation behavior in the two-electron momentum distributions along the polarization direction. For Ar emission into opposite hemispheres dominates ("anticorrelation," ACO) whereas for Ne the electrons predominantly emerge into the same hemisphere ("correlation," CO). Both can be partly explained within threedimensional (3D) classical calculations including quantum effects, like tunneling of the second electron. Inspecting the transverse momenta we shed light on the dynamics, investigate Coulomb focusing effects, find nonclassical dynamics, and make predictions about threshold intensities, as well as CO- and ACO-dominated regimes.
NSDI essentially occurs in the so-called tunneling regime and is usually explained within the quasiclassical recollision model [5]. Here, the first electron is set free (tunnels) near the maximum of laser field, accumulates energy during about half a laser cycle (up to $3.17 U_{P}$ ), is thrown back on its parent ion near a zero crossing of the laser field and, in a collision, ejects the second electron. Both emitted electrons prefer to move into the same hemisphere along the laser polarization axis [6-11], dubbed correlation (CO). Even though this general scenario is well established, distinct differences do occur for different target species; i.e., significant structure dependence is observed. Up to now, this is qualitatively explained via target dependent cross sections for (recolliding) electron-impact excitation of the ionic core. If this cross section is small, direct recollision ionization dominates with both electrons being mainly ejected into the same hemisphere. Is it large, as, e.g., for $\operatorname{Ar} 3 s$ to $3 p$ excitation, recollision-induced excitation with subsequent field ionization [7] becomes important, with both electrons being equally likely emitted in the same or opposite hemispheres. However, this discussion cannot be considered completely settled, since other ionization mechanisms leading to anticorrelation at high intensities, close to the over-barrier-ionization regime, have been discussed recently [12].

Even less clear is the situation at lower intensities, approaching the so-called recollision threshold where, in a classical picture, the returning electron's energy is too small to overcome the field-suppressed ionization or even excitation potential of the parent ion. Only recently, few experiments have addressed this fundamental threshold regime dominated by multiphoton-multielectron interactions $[11,13,14]$. Penetrating deep into the MPI regime for 
Ar back-to-back emission (so-called "anticorrelation") was found for the first time ever to dominate strong-field DI [3]. Comparing with predictions of quantum [15] as well as classical calculations it was concluded that multiple, inelastic field-assisted recollisions, resonantly exciting the target ion, were responsible for the observation. More recently quantum calculations [16] have addressed this regime. Exclusively considering transition amplitudes where both electrons are ejected simultaneously, emission into the same hemisphere was found to dominate for Ar. Dedicated classical model calculation [17] on the other hand, considering the contributions from multiple recollisions and, in addition, implementing tunneling of the second electron [4], clearly revealed the back-to-back emission characteristics observed in the experiment. Whereas in [17], the anticorrelated electrons were found to be mainly produced via multiple collisions in the first or second field maximum after recollision, the analysis in [4] yields a more subtle picture. Here, the contributions to anticorrelated electron emission due to classical multiple recollision induced direct-ionization (REDI) can be distinguished from those due to recollision induced excitation plus tunneling (RIET) of the second electron. Whereas the REDI contribution to correlated emission decreases with decreasing intensity, the RIET part is expected to increase. For anticorrelated events, instead, both mechanisms contribute when lowering the intensity causing ACO to dominate over CO deep in the MPI regime in agreement with experiment. Even though the latter calculations draw a quite detailed picture, the problem can certainly not be considered being solved, since quantum theories are widely lacking and the dependence on the target structure, expected to be very important for the case of excitationtunneling ionization, has not been investigated at all.

Experimentally, the coincident detection of electron and ions in the MPI regime is challenging due to the exceedingly low DI yield. The major breakthrough enabling such measurements was the demonstration of a high-power (peak power $\sim 16 \mathrm{MW}$ ), long-cavity femtosecond (46 fs) laser system reaching a repetition rate of $6 \mathrm{MHz}$ and pulse intensities up to $2 \times 10^{14} \mathrm{~W} / \mathrm{cm}^{2}$ [18]. The coincidence method, the dedicated reaction microscope (REMI), momentum resolutions achieved as well as the intensity calibration procedure and accuracy are described in detail elsewhere [19]. In the REMI, ions and electrons were projected onto two position-sensitive detectors by weak electric $(2 \mathrm{~V} / \mathrm{cm})$ and magnetic $(4.5 \mathrm{G})$ fields applied along the laser polarization axis. All experiments were performed at an ion rate below $2 \times 10^{-5}$ per laser pulse (target density: $1 \times 10^{12} / \mathrm{cm}^{3}$ ) such that all measured electrons and ions can be safely related to the fragmentation of a single atom in the focus (diameter $\sim 1 \mu \mathrm{m}$ ). Under present settings, data collection for each target took several weeks.

We recorded a total of 1780 double coincidence events (ion and one electron) for $\mathrm{Ne}^{2+}$ with a ratio of $\mathrm{Ne}^{2+} / \mathrm{Ne}^{+} \sim 1.5 \times 10^{-4}$ at an intensity of $1.5 \times$ $10^{14} \mathrm{~W} / \mathrm{cm}^{2}$ and 1160 counts for Ar at $3 \times 10^{13} \mathrm{~W} / \mathrm{cm}^{2}$ with $\mathrm{Ar}^{2+} / \mathrm{Ar}^{+} \sim 8 \times 10^{-4}$. To possibly even further penetrate into the MPI regime we continued to decrease the laser intensity to about $1.5 \times 10^{13} \mathrm{~W} / \mathrm{cm}^{2}$ (Ar) and $8 \times 10^{13} \mathrm{~W} / \mathrm{cm}^{2}(\mathrm{Ne})$. Here, however, we could not find any indication of DI events while running the measurements for several weeks for each species. This means that DI rates decrease by at least 3 orders of magnitude between $1.5 \times 10^{14}\left(3 \times 10^{13}\right) \mathrm{W} / \mathrm{cm}^{2}$ and $8 \times 10^{13}(1.5 \times$ $\left.10^{13}\right) \mathrm{W} / \mathrm{cm}^{2}$ for neon (argon).

In Fig. 1 we present $P_{\|}$-correlation plots between both electrons (left column) and electron transverse momentum $\left(P_{\perp}\right)$ distributions (right column) at intensities of $3 \times$ $10^{13} \mathrm{~W} / \mathrm{cm}^{2}$ (Ar, upper row) and $1.5 \times 10^{14} \mathrm{~W} / \mathrm{cm}^{2}$ (Ne, lower row), i.e., at the lowest intensities ever investigated. Even though the absolute single ionization count rate is rather comparable for those targets, both spectra, the correlation maps as well as $P_{\perp}$ distributions show distinctly different shapes. Whereas for Ar a substantial amount of anticorrelated electrons are observed in line with our previous measurements at slightly higher intensity [3] correlated emission is found to dominate in case of Ne. Differences in the dynamics become especially obvious if one inspects the transverse momenta of electrons coincident to double ionization (solid curve, right row) with a cusplike structure for Ar that is substantially broadened in the case of Ne. Even taking into account an at least 30\% uncertainty in the absolute determination of the intensity and, thus, thinking we might be deeper in the MPI regime for Ar than for $\mathrm{Ne}$, we still find similarly distinct differences if we compare the $\mathrm{Ne}$ spectrum to the one for $\mathrm{Ar}$ at $7 \times 10^{13} \mathrm{~W} / \mathrm{cm}^{2}$ [dashed line in Fig. 1(b)].

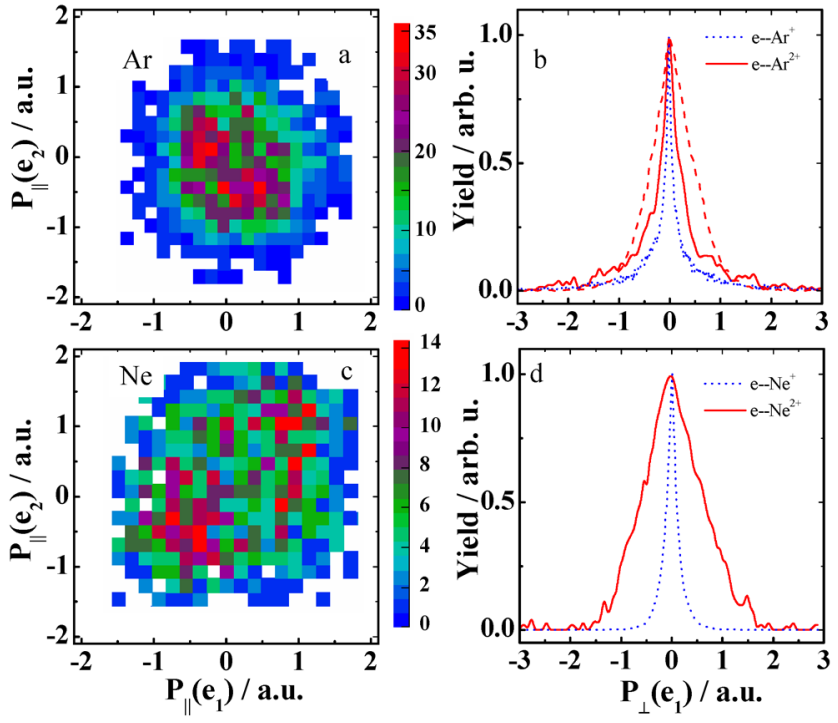

FIG. 1 (color online). Parallel momentum correlation of two electrons for argon (a) $\left(3 \times 10^{13} \mathrm{~W} / \mathrm{cm}^{2}\right)$ and neon (c) $\left(1.5 \times 10^{14} \mathrm{~W} / \mathrm{cm}^{2}\right)$. (b) Transverse momentum distributions of electrons in coincidence with $\mathrm{Ar}^{1+}$ (dotted line) and $\mathrm{Ar}^{2+}$ at $3 \times 10^{13} \mathrm{~W} / \mathrm{cm}^{2}$ (solid line) and at $7 \times 10^{13} \mathrm{~W} / \mathrm{cm}^{2}$ (dashed solid line) as well as (d) for $\mathrm{Ne}^{1+}$ and $\mathrm{Ne}^{2+}$ at $1.5 \times$ $10^{14} \mathrm{~W} / \mathrm{cm}^{2}$. 
The striking experimental structure dependence can be partially explained by our semiclassical model, in which the RIET effect has been taken into account within the Wentzel-Kramers-Brillouin (WKB) approach [4]. The energy level of the first excitation state of $\mathrm{Ne}^{+}$and the fieldfree DI threshold are both significantly higher than those of $\mathrm{Ar}^{+}$. It is thus much more difficult to resonantly excite the second electron during recollision in a correct quantummechanical description. To take this effect approximately into account in our classical model, we have abandoned all DI trajectories for $\mathrm{Ne}$ if the inner electron is excited to an energy that is lower than the first excited state. Numerical results are shown in Figs. 2(a) and 2(c) and the correlated parallel momentum distributions for $\mathrm{Ar}$ and $\mathrm{Ne}$ are in quite good qualitative agreement with the observations, exhibiting $\mathrm{ACO}$ for $\mathrm{Ar}$ and $\mathrm{CO}$ for $\mathrm{Ne}$, respectively.

Inspecting the electron transverse momentum distributions for single ionization and DI shown in Figs. 1(b) and 1(d) for the experiment as well as in Figs. 2(b) and 2(d) for our model calculations we find in general pronounced "cusplike" structures as observed before. It has been proposed that Coulomb focusing [20] or the Coulomb singularity [21] are responsible for their formation for single ionization in accordance with the present data [dotted curves in Figs. 1(b), 1(d), 2(b), and 2(d)]. Whereas the experimental momentum distribution of electrons coincident with $\mathrm{Ar}^{2+}$ ions (solid line) also shows a clear cusplike shape, only slightly broader than for single ionization and in good agreement with theory the respective distribution for $\mathrm{Ne}$ is significantly broader than predicted. In general, the cusplike shape is obtained as a result of the singularity in the electron continuum wave function at zero energy in the presence of a Coulomb potential [20]. Thus, we conclude that for Ar double ionization both electrons have very little energy in the final state. This is expected within the
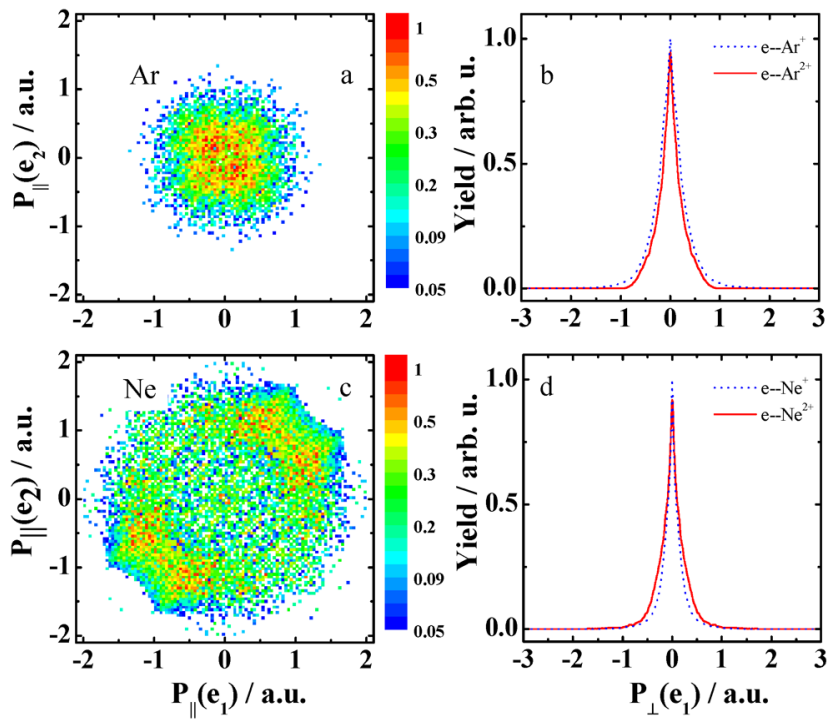

FIG. 2 (color online). Same as Fig. 1 but for theoretical calculations (see text).
RIET picture, where the second electron tunnels, being essentially set into the continuum with zero momentum, independently from the first electron. Accordingly, our model calculations [Fig. 2(b)] largely reproduce the experimental data in Fig. 1(b).

On the other hand, electrons emerging coincident to $\mathrm{Ne}^{2+}$ production are observed to receive much larger transverse momenta and show a more Gaussian-like shape. This indicates that the recollision mechanism still dominates, where the recolliding electron could be scattered to a larger angle with its longitudinal momentum being transferred to the transverse direction. Surprisingly, such a feature is not found in our model calculations where the transverse momentum distribution of an electron coincident to the simulated $\mathrm{Ne}^{2+}$ ion, excluding trajectories that lead to unphysical low excitation energies, is shown in Fig. 2(d). Obviously such a model does not capture the full quantum dynamics of the system in all of its aspects correctly, underlining the quest for quantum calculations.

Finally, we investigate and predict the threshold behavior which has been previously addressed theoretically only for DI of $\mathrm{He}[22,23]$. When considering the full 3D Schrödinger equation of a one-electron atom in a uniform field in the framework of parabolic coordinates [24], the $3 \mathrm{D}$ problem can be reduced to $1 \mathrm{D}$ with an effective potential of $U(\eta)=-1 / 2 \eta-1 / 8 \eta^{2}-\varepsilon_{0} \eta / 8$. Here, $\eta=r-z$ is the parabolic coordinate representing the distance $r$ of the electron to core minus its coordinate in the field direction $z$, and $\varepsilon_{0}$ is the field strength. There exists a potential barrier along the $\eta$ coordinate for ionization of the electron with $z \rightarrow+\infty$, corresponding to its passage into the region of large $\eta$. Ignoring the slight influence from the second term in the above effective potential, we can readily estimate the maximum of the barrier as $-\sqrt{\varepsilon_{0}} / 2$. On the other side, the maximum effective total energy that will be shared by the two electrons after recollision close to a zero crossing of the field is $\left(-\left|I_{P 2}(t)\right|+3.17 U_{p}\right) / 4$ [where $I_{P 2}(t)$ is the ionization potential of inner electron and the factor 1/4 arises from the transformation from Cartesian to parabolic coordinates]. DI as a result of a single recollision event becomes possible only when both electrons can pass over the suppressed barrier, with the solution depending on the energy be sharing between the electrons after recollision. By making an average on two limiting cases that correspond to equal and most unequal energy partition, we can estimate the boundary for the transition to ACO to be around $3.17 U_{p}-\left|I_{P 2}(t)\right|=-3 \sqrt{\varepsilon_{0}}$ (see [4] for details). Above that limit, the DI events are mainly induced by single recollision and, therefore, $\mathrm{CO}$ dominates.

In the same spirit, we estimate a criterion $3.17 U_{p}-$ $\left|I_{e}(t)\right|=-2 \sqrt{\varepsilon_{0}}$ where DI cutoff should occur, where $I_{e}$ is the first excitation energy of the parent ion. Below that threshold, the maximum recollision energy is not enough to excite the bounded electron to at least the first excited state while the returning electron keeps just the minimum energy to escape. Therefore, DI is expected to decrease 


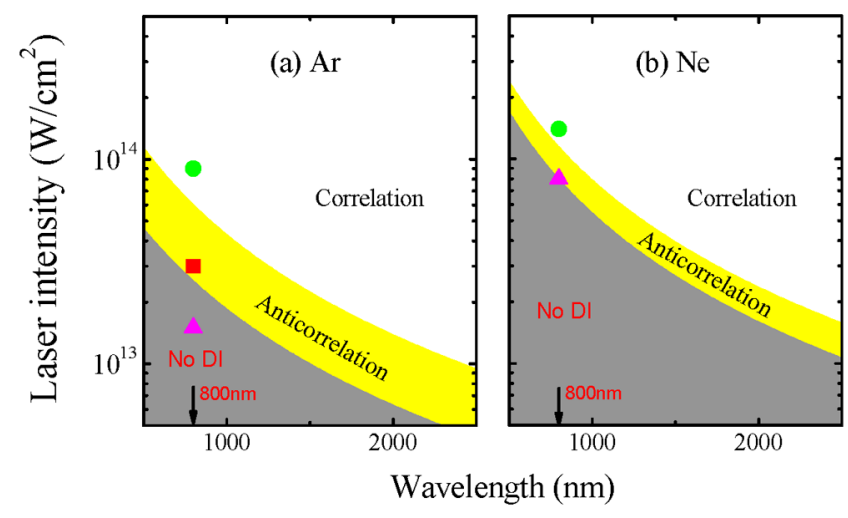

FIG. 3 (color online). Predicted areas where correlated or anticorrelated electron emission dominates as well as the boundary below which DI should be forbidden at all. Points indicate the present measurement where correlation (circle), anticorrelation (square), and no DI (triangle) have been observed, respectively.

sharply. The results are plotted in Fig. 3 for both, Ar and Ne together with the experimental data finding overall agreement. Especially, the ACO zone predicted by our theory for $\mathrm{Ne}$ is extremely narrow and, thus, might hardly be observable in experiments.

In conclusion, we have presented kinematically complete measurements and model calculations for single and double ionization of $\mathrm{Ar}$ and $\mathrm{Ne}$ in infrared laser fields in the DI threshold regime, at the lowest intensities ever investigated. Whereas for Ar anticorrelation in the longitudinal two-electron momentum spectra is observed in agreement with previous measurements at slightly higher intensity, correlation dominates for the Ne target similarly close to the threshold. Further lowering the intensity led to the complete absence of any double ionization event over weeks of data taking time. We can reproduce that salient feature in the correlation plot with 3D classical calculations including tunneling for the second electron if, for the $\mathrm{Ne}$ target, all trajectories are rejected with recollision energies below the first excitation level of $\mathrm{Ne}^{+}$. This provides strong evidence that the target structure, i.e., the low-lying excited levels in $\mathrm{Ar}^{+}$along with the large electron-impact excitation cross section are the reason for this observation. We have further made predictions on the regimes where one would expect correlation and anticorrelation behavior for both targets and provide a classical threshold below which DI should not occur in good agreement with the experimental results. From that it might be concluded (i) that we have really approached the DI threshold and (ii) that it might be impossible, at least very difficult to observe ACO for the Ne target since the allowed intensity range is very small.

Inspecting the transverse momentum distribution of electrons coincident with singly and doubly charged $\mathrm{Ne}$ and $\mathrm{Ar}$ ions, we shed light on the dynamics and provide clear evidence that our essentially classical calculations do not capture all of the dynamics: Whereas the cusplike behavior for single ionization is in good agreement with the experimental data, the experimental transverse electron momentum distribution for $\mathrm{Ne}^{2+}$ is significantly broader as predicted pointing to the existence of correlated quantum motion not taken into account by the classical model and underlining the quest for many-particle quantum approaches.

Y.L. thanks the support of "Bairen" program at PKU and NCET. This work is partially supported by the 973 projects (2007CB814800, 2007CB815103, and 2006CB806007) and NSFF (10725521 and 10821062).

\footnotetext{
*Yunquan.liu@pku.edu.cn

${ }^{\dagger}$ Liu_jie@iapcm.ac.cn

+qhgong@pku.edu.cn

${ }^{\S}$ Joachim.Ullrich@mpi-hd.mpg.de
}

[1] D. N. Fittinghoff et al., Phys. Rev. Lett. 69, 2642 (1992); B. Walker et al., Phys. Rev. Lett. 73, 1227 (1994).

[2] A. Becker, R. Dörner, and R. Moshammer, J. Phys. B 38, S753 (2005).

[3] Y. Liu et al., Phys. Rev. Lett. 101, 053001 (2008).

[4] D.-F. Ye and J. Liu, Phys. Rev. A 81, 043402 (2010).

[5] K. Schafer et al., Phys. Rev. Lett. 70, 1599 (1993); P. B. Corkum, Phys. Rev. Lett. 71, 1994 (1993); See also M. Yu. Kuchiev, JETP Lett. 45, 404 (1987).

[6] A. Rudenko et al., Phys. Rev. Lett. 93, 253001 (2004).

[7] B. Feuerstein et al., Phys. Rev. Lett. 87, 043003 (2001).

[8] Th. Weber et al., Nature (London) 405, 658 (2000).

[9] R. Moshammer et al., Phys. Rev. Lett. 84, 447 (2000).

[10] J. Parker et al., Phys. Rev. Lett. 96, 133001 (2006); A. Rudenko et al., Phys. Rev. Lett. 99, 263003 (2007); A. Staudte et al., Phys. Rev. Lett. 99, 263002 (2007).

[11] E. Eremina et al., J. Phys. B 36, 3269 (2003).

[12] A. Emmanouilidou and A. Staudte, arXiv:0909.3409v1.

[13] R. Wiehle and B. Witzel, Phys. Rev. Lett. 89, 223002 (2002).

[14] J. L. Chaloupka et al., Phys. Rev. Lett. 90, 033002 (2003).

[15] J. S. Prauzner-Bechcicki et al., Phys. Rev. Lett. 98, 203002 (2007).

[16] D. I. Bondar, W.-K. Liu, and M. Yu. Ivanov, Phys. Rev. A 79, 023417 (2009).

[17] S. L. Haan et al., J. Phys. B 41, 211002 (2008).

[18] S. Dewald et al., Opt. Lett. 31, 2072 (2006); Y. Liu et al., Opt. Express 15, 18103 (2007).

[19] V.L. B. de Jesus et al., J. Phys. B 37, L161 (2004).

[20] D. Comtois et al., J. Phys. B 38, 1923 (2005).

[21] A. Rudenko et al., J. Phys. B 38, L191 (2005).

[22] B. Sheehy et al., Phys. Rev. A 58, 3942 (1998).

[23] H. W. van der Hart and K. Burnett, Phys. Rev. A 62, 013407 (2000).

[24] Quantum Mechanics, edited by D. Landau and E. M. Lifshitz (Pergamon Press, New York, 1977), 3rd ed., Chap. V. Here, we take the parameters $\beta_{1}=\beta_{2}=1$. This is because we are dealing with a two-electron heliumlike atom in which the nuclear charge $Z=2$ and, thus, $\beta_{1}+\beta_{2}=2$. Note that $\beta_{1}=\beta_{2}=\frac{1}{2}$ is chosen for hydrogen in the book. See also Ref. [4] for more details. 